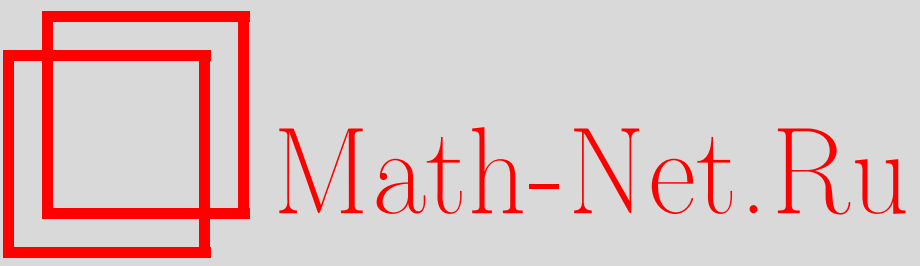

Ж. И. Абдуллаев, Связанные состояния системы двух фермионов на одномерной решетке, ТМФ, 2006, том 147, номер 1, 47-57

DOI: https://doi.org/10.4213/tmf2021

Использование Общероссийского математического портала Math-Net.Ru подразумевает, что вы прочитали и согласны с пользовательским соглашением

http://www.mathnet.ru/rus/agreement

Параметры загрузки:

IP: 3.89 .197 .203

26 апреля 2023 г., 18:04:45 
ТЕОРЕТИЧЕСКАЯ

И МАТЕМАТИЧЕСКАЯ

ФИЗИКА

Том 147, № 1

апрель, 2006

(C) 2006 г.

Ж. И. Абдуллаев*

\section{СВЯЗАННЫЕ СОСТОЯНИЯ СИСТЕМЫ ДВУХ ФЕРМИОНОВ НА ОДНОМЕРНОЙ РЕШЕТКЕ}

Рассматривается гамильтониан системы двух фермионов на одномерной целочисленной решетке. Доказано, что число связанных состояний $N(k)$ - неубывающая функция от полного квазиимпульса системы $k \in[0, \pi]$. Описано множество точек разрыва функции $N(k)$ и вычислен скачок $N(k+0)-N(k)$ в точках разрыва. Установлено, что энергия связанного состояния $z_{n}(k)$ возрастает при возрастании полного квазиимпульса $k \in[0, \pi]$.

Ключевые слова: гамильтониан, связанное состояние, полный квазиимпульс, оператор Шредингера, собственное значение, резонанс, принцип Бирмана-Швингера.

\section{1. ВВЕДЕНИЕ}

Дискретный спектр двухчастичного непрерывного оператора Шредингера

$$
h_{\lambda}=-\Delta+\lambda V
$$

исследовался многими авторами, причем условия на потенциал $V$ формулировались в его координатном представлении. Условия конечности отрицательного спектра и отсутствия положительных собственных значений оператора $h_{\lambda}$ приведены в [1]. Если $V \leqslant 0$, то число отрицательных собственных значений $N(\lambda)$ есть неубывающая функция от $\lambda \in(0, \infty)$ и каждое собственное значение $z_{n}(\lambda)$ убывает в диапазоне $(0, \infty)$. Известно, что с уменьшением константы связи $\lambda$ значения энергии связанных состояний оператора $h_{\lambda}$ приближаются к краю непрерывного спектра (см. [1]) и при некотором конечном значении $\lambda$ попадают на край. При этом возникают два вопроса: соответствуют ли такому пороговому состоянию связанное или виртуальное состояние (т.е. квадратично-интегрируема ли соответствующая волновая функция) и куда исчезают связанные состояния при дальнейшем уменьшении $\lambda$ ? Изучению первого вопроса посвящены работы Рауха [2], Саймона [3], Клауза [4]. Что касается второго вопроса, оказывается, что связанное состояние исчезает, поглощаясь непрерывным спектром, и превращается в резонанс. При этом резонанс трактуется как полюс аналитического продолжения резольвенты через разрезы непрерывного

*Самаркандский государственный университет, Самарканд, Узбекистан. E-mail: jabdullaev@mail.ru 
спектра и может быть физическим и нефизическим. Физическому резонансу соответствует пик в амплитуде рассеяния. Доказательству этой картины посвящены работы Рауха [2] и Хауленда [5].

Природа появления связанных состояний двухчастичных кластерных операторов при малых значениях параметра кластерности впервые подробно исследовалась Маматовым и Минлосом [6], а потом в более общей ситуации - Минлосом и Могильнером [7]. Хауленд [5] показал, что теорема Реллиха о возмущении собственных значений не распространяется на теорию резонансов.

Резонансы для краевых задач с одномерным уравнением Шредингера впервые возникли в работе Редже [8]. Удобный и плодотворный подход к исследованию процессов резонансного рассеяния был развит Лаксом и Филлипсом [9].

Исследование связанных состояний гамильтониана $H$ системы двух фермионов на одномерной решетке сводится к изучению собственных значений семейства операторов Шредингера $H(k), k \in \mathbf{T} \equiv(-\pi, \pi]$ (см. ниже $(2.2))$. Поэтому мы изучим собственные значения оператора $H(k)$. Докажем, что число собственных значений $N(k)$ оператора $H(k)$ есть неубывающая функция от $k \in[0, \pi]$, где $k$ - полный квазиимпульс системы. В предположении, сформулированном в разделе 4, каждое собственное значение $z_{n}(k)$ возрастает при возрастании $k \in[0, \pi]$. Из равенства $H(-k)=H(k)$ (см. $(2.2))$ следует, что $N(-k)=N(k)$ и $z_{n}(-k)=z_{n}(k)$. Поэтому в дальнейшем считаем, что $k \in[0, \pi]$. Функция $N(k)$ кусочно-постоянна и непрерывна слева. Точки разрыва $N(k)$ суть решения уравнения $\omega(k)=4 \mu_{n}, n \in \mathbb{N}$, где $\mu_{n}-$ различные собственные значения положительного компактного оператора $G(0)$ (см. ниже $(3.11)), \omega(k)$ - ширина непрерывного спектра оператора $H(k)$.

\section{2. ОПИСАНИЕ ДВУХЧАСТИЧНОГО ГАМИЛЬТОНИАНА}

Свободному гамильтониану $\widehat{H}_{0}$ системы двух фермионов на одномерной решетке $\mathbb{Z}$ обычно соответствует ограниченный самосопряженный оператор, действующий в гильбертовом пространстве $\ell_{2}^{\text {as }}\left(\mathbb{Z}^{2}\right)=\left\{f \in \ell_{2}\left(\mathbb{Z}^{2}\right): f(n, m)=-f(m, n)\right\}$ по формуле

$$
\widehat{H}_{0}=-\frac{1}{2 m} \Delta_{x_{1}}-\frac{1}{2 m} \Delta_{x_{2}} .
$$

Здесь $m$ - масса фермиона, $\Delta_{x_{1}}=\Delta \otimes I$ и $\Delta_{x_{2}}=I \otimes \Delta$, где решетчатый лапласиан $\Delta$ есть разностный оператор, описывающий перенос частицы с узла на соседний узел, т.е.

$$
(\Delta \hat{\psi})(x)=\hat{\psi}(x+1)-2 \hat{\psi}(x)+\hat{\psi}(x-1), \quad \hat{\psi} \in \ell_{2}(\mathbb{Z}) .
$$

Полный гамильтониан $\widehat{H}$ действует в гильбертовом пространстве $\ell_{2}^{\text {as }}\left(\mathbb{Z}^{2}\right)$ и представляет собой разность свободного гамильтониана $\widehat{H}_{0}$ и потенциала взаимодействия $\widehat{V}_{2}$ двух частиц (см. [10]):

$$
\widehat{H}=\widehat{H}_{0}-\widehat{V}_{2}
$$

где

$$
\left(\widehat{V}_{2} \hat{\psi}\right)\left(x_{1}, x_{2}\right)=\hat{v}\left(x_{1}-x_{2}\right) \hat{\psi}\left(x_{1}, x_{2}\right), \quad \hat{\psi} \in \ell_{2}\left(\mathbb{Z}^{2}\right) .
$$

Всюду в дальнейшем относительно функции $\hat{v}$ предполагается, что

$$
\hat{v}(x)=\hat{v}(-x) \geqslant 0, \quad x \in \mathbb{Z}, \quad \lim _{|x| \rightarrow \infty}|x|^{\alpha} \hat{v}(x)=0, \quad \alpha>2 .
$$


При выполнении условия (2.1) оператор $\hat{V}_{2}$ является ограниченным и самосопряженным в пространстве $\ell_{2}^{\text {as }}\left(\mathbb{Z}^{2}\right)$. Отсюда следует ограниченность и самосопряженность гамильтониана $\widehat{H}$.

Переход в импульсное представление осуществляется с помощью преобразования Фурье $\mathcal{F}: \ell_{2}\left(\mathbb{Z}^{2}\right) \longrightarrow L_{2}\left(\mathbf{T}^{2}\right)$, где $L_{2}\left(\mathbf{T}^{2}\right) \equiv L_{2}(\mathbf{T}) \otimes L_{2}(\mathbf{T}), L_{2}(\mathbf{T})$ - гильбертово пространство функций, определенных на $\mathbb{R}=(-\infty, \infty)$ с периодом $2 \pi$ и квадратичноинтегрируемых на $\mathbf{T} \equiv(-\pi, \pi]$. Заметим, что $\mathcal{F}\left(\ell_{2}^{\text {as }}\left(\mathbb{Z}^{2}\right)\right)=L_{2}^{\text {as }}\left(\mathbf{T}^{2}\right) \equiv\left\{f \in L_{2}\left(\mathbf{T}^{2}\right)\right.$ : $\left.f\left(k_{1}, k_{2}\right)=-f\left(k_{2}, k_{1}\right)\right\}$.

Гамильтониан $H$ системы двух фермионов действует как ограниченный самосопряженный оператор в гильбертовом пространстве $L_{2}^{\mathrm{as}}\left(\mathbf{T}^{2}\right)$ и имеет вид

$$
H=\mathcal{F} \widehat{H} \mathcal{F}^{-1} \equiv H_{0}-V_{2} .
$$

Свободный гамильтониан $H_{0}$ - это оператор умножения в $L_{2}^{\text {as }}\left(\mathbf{T}^{2}\right)$ на функцию $\varepsilon\left(k_{1}\right)+\varepsilon\left(k_{2}\right)$, где $\varepsilon(q)=1-\cos q$ есть энергия отдельного фермиона с импульсом $q \in \mathbf{T}$. Оператор взаимодействия $V_{2}$ двух фермионов действует в $L_{2}^{\mathrm{as}}\left(\mathbf{T}^{2}\right)$ по формуле

$$
\left(V_{2} f\right)\left(k_{1}, k_{2}\right)=(2 \pi)^{-1 / 2} \int_{\mathbf{T}^{2}} v\left(k_{1}-k_{1}^{\prime}\right) \delta\left(k_{1}+k_{2}-k_{1}^{\prime}-k_{2}^{\prime}\right) f\left(k_{1}^{\prime}, k_{2}^{\prime}\right) d k_{1}^{\prime} d k_{2}^{\prime},
$$

где $\delta(k)$ - дельта-функция Дирака, $v$ - преобразование Фурье функции $\hat{v}$. Из условия $(2.1)$ следует, что функция $v(q)$ является четной, вещественнозначной, непрерывной функцией на $\mathbf{T}$. Полный гамильтониан $H$ коммутирует с группой унитарных операторов $U_{s}, s \in \mathbb{Z}$ :

$$
\left(U_{s} f\right)\left(k_{1}, k_{2}\right)=e^{-i s\left(k_{1}+k_{2}\right)} f\left(k_{1}, k_{2}\right), \quad f \in L_{2}\left(\mathbf{T}^{2}\right) .
$$

Отсюда следует (см. [1]), что существуют разложения пространства $L_{2}^{\text {as }}\left(\mathbf{T}^{2}\right)$, а также операторов $U_{s}$ и $H$ в прямые интегралы:

$$
L_{2}^{\mathrm{as}}\left(\mathbf{T}^{2}\right)=\int_{\mathbf{T}} \bigoplus L_{2}^{\mathrm{as}}\left(\mathcal{F}_{k}\right) d k, \quad U_{s}=\int_{\mathbf{T}} \bigoplus U_{s}(k) d k, \quad H=\int_{\mathbf{T}} \bigoplus \widetilde{H}(k) d k .
$$

Здесь

$$
\mathcal{F}_{k}=\left\{\left(k_{1}, k_{2}\right) \in \mathbf{T}^{2}: k_{1}+k_{2}=k, k \in \mathbf{T}\right\},
$$

$U_{s}(k)$ - оператор умножения на число $e^{-i s k}$ в пространстве $L_{2}^{\text {as }}\left(\mathcal{F}_{k}\right)$. Слойный оператор $\widetilde{H}(k)$ также действует в $L_{2}^{\text {as }}\left(\mathcal{F}_{k}\right)$ по формуле

$$
(\widetilde{H}(k) f)(q, k-q)=(\varepsilon(q)+\varepsilon(k-q)) f(q, k-q)-(2 \pi)^{-1 / 2} \int_{\mathbf{T}} v(q-s) f(s, k-s) d s
$$

и унитарно эквивалентен оператору $H(k)$, действующему в гильбертовом пространстве $L_{2}^{-}(\mathbf{T}) \equiv\left\{f \in L_{2}(\mathbf{T}): f(-p)=-f(p)\right\}$ по формуле

$$
(H(k) f)(q)=\left(2-2 \cos \frac{k}{2} \cos q\right) f(q)-(2 \pi)^{-1 / 2} \int_{\mathbf{T}} v(q-s) f(s) d s .
$$

Эквивалентность осуществляется с помощью унитарного преобразования

$$
u_{k}: L_{2}^{\mathrm{as}}\left(\mathcal{F}_{k}\right) \rightarrow L_{2}^{-}(\mathbf{T}), \quad\left(u_{k} g\right)(q)=g\left(\frac{k}{2}-q, \frac{k}{2}+q\right) .
$$


Оператор $H(k)=H_{0}(k)-V$ называется оператором Шредингера, $H_{0}(k)$ есть оператор умножения на функцию $\varepsilon_{k}(q)=2-2 \cos (k / 2) \cos q, V$ - интегральный оператор в $L_{2}^{-}(\mathbf{T})$, порожденный ядром $(2 \pi)^{-1 / 2} v(q-s)$. При условии $(2.1)$ оператор $V$ положителен и его положительный квадратный корень $V^{1 / 2}$ принадлежит классу со следом $\Sigma_{1}$. Чтобы избежать конечномерности возмущения предположим, что

$$
\operatorname{card}\{n \in \mathbb{Z}: \hat{v}(n)>0\}=\infty \text {. }
$$

\section{3. СВЯЗАННЫЕ СОСТОЯНИЯ СИСТЕМЫ ДВУХ ФЕРМИОНОВ}

Приведем некоторые известные факты и обозначения. Для любого самосопряженного оператора $B$, действующего в гильбертовом пространстве $\mathcal{H}$ и не имеющего существенного спектра правее точки $\mu \in \mathbb{R}$, обозначим через $\mathcal{H}_{B}(\mu) \subset \mathcal{H}$ подпространства такие, что ненулевые элементы $f \in \mathcal{H}_{B}(\mu)$ удовлетворяют неравенству $(B f, f)>\mu(f, f)$, и положим

$$
n(\mu, B)=\sup _{\mathcal{H}_{B}(\mu)} \operatorname{dim} \mathcal{H}_{B}(\mu)
$$

Число $n(\mu, B)$ совпадает с числом собственных значений оператора $B$, лежащих правее точки $\mu$. Если для любого ненулевого $f \in \mathcal{H}$ выполняется неравенство $(B f, f)>$ 0 , то мы пишем $B>0$.

Спектр невозмущенного оператора $H_{0}(k)$ чисто непрерывный и совпадает с отрезком $[m(k), M(k)]$, где

$$
m(k)=\min _{p \in[-\pi, \pi]} \varepsilon_{k}(p)=2-2 \cos \frac{k}{2}, \quad M(k)=\max _{p \in[-\pi, \pi]} \varepsilon_{k}(p)=2+2 \cos \frac{k}{2} .
$$

Длина этого отрезка $\omega(k)=M(k)-m(k)=4 \cos (k / 2)$ называется шириной непрерывного спектра оператора $H(k)$. Ширина $\omega(k)$ есть четная функция, убывающая на интервале $[0, \pi]$. Она обращается в нуль, когда полный квазиимпульс $k$ принимает максимальное значение $\pi$. В этом случае оператор $H(\pi)=2 I-V$ (см. (2.2)) имеет собственные значения вида $z_{n}(\pi)=2-\hat{v}(n), \quad n \in \mathbb{N}$. Собственному значению $z_{n}(\pi), \quad n \in \mathbb{N}$, соответствует нормированная собственная функция

$$
\varphi_{n}(p)=\frac{\sin (n p)}{\sqrt{\pi}}, \quad n \in \mathbb{N} .
$$

В силу теоремы Гильберта-Шмидта ядро $(2 \pi)^{-1 / 2} v(p-q)$ интегрального оператора $V$ представляется в виде

$$
(2 \pi)^{-1 / 2} v(p-q)=\sum_{n=1}^{\infty} \hat{v}(n) \varphi_{n}(p) \varphi_{n}(q) .
$$

Из самосопряженности оператора $H(k)$ и положительности оператора $V$ вытекает, что $\sigma(H(k)) \cap(M(k), \infty)=\varnothing$, поэтому $\sigma_{\text {disc }}(H(k)) \subset(-\infty, m(k))$. Через $N(k, z)$ обозначается число собственных значений оператора $H(k)$, лежащих левее точки $z \leqslant$ $m(k)$, т.е. $N(k, z)=n(-z,-H(k))$. Для простоты $N(k, m(k))$ обозначим через $N(k)$. 
Число $N(k)$ фактически совпадает с числом собственных значений вне непрерывного спектра оператора $H(k)$.

Для любого $k \in \mathbf{T}$ и $z<m(k)$ мы определим интегральные операторы

$$
G(k, z)=V^{1 / 2} r_{0}(k, z) V^{1 / 2}, \quad Q(k, z)=r_{0}^{1 / 2}(k, z) V^{1 / 2},
$$

где $r_{0}(k, z)$ - резольвента невозмущенного оператора $H_{0}(k)$. При условии $(2.1)$ оператор $V^{1 / 2}$ принадлежит классу со следом $\Sigma_{1}$, следовательно, $Q(k, z) \in \Sigma_{1}$. Из представления $G(k, z)=(Q(k, z))^{*} Q(k, z)$ следует его положительность и принадлежность классу $\Sigma_{1}$ при всех $k \in \mathbf{T}$ и $z<m(k)$. Кроме того, имеет место принцип Бирмана-Швингера

$$
N(k, z)=n(1, G(k, z)) \quad \text { при всех } \quad z<m(k) .
$$

Решение $f$ уравнения Шредингера

$$
H(k) f=z f
$$

и неподвижные точки $\varphi$ оператора $G(k, z)$ связаны соотношениями

$$
f=r_{0}(k, z) V^{1 / 2} \varphi, \quad \varphi=V^{1 / 2} f .
$$

ЛЕмма 1. Число $z<m(k)$ является собственным значением оператора $H(k)$ тогда и только тогда, когда число $\lambda=1$ есть собственное значение оператора $G(k, z)$.

ДокАЗАтЕЛьСтво. Пусть ненулевое значение $f \in L_{2}^{-}(\mathbf{T})$ является решением уравнения (3.3). Введя обозначение $\varphi=V^{1 / 2} f$, из (3.3) получим $f=r_{0}(k, z) V^{1 / 2} \varphi$, следовательно, $V^{1 / 2} f=V^{1 / 2} r_{0}(k, z) V^{1 / 2} \varphi$. Учитывая $\varphi=V^{1 / 2} f$, мы получим, что уравнение

$$
\varphi=G(k, z) \varphi
$$

имеет ненулевое решение. Обратно, если уравнение (3.5) имеет ненулевое решение $\varphi \in L_{2}^{-}(\mathbf{T})$, то функция $f$, построенная в (3.4), отлична от нуля, удовлетворяет уравнению Шредингера (3.3) и принадлежит $L_{2}^{-}(\mathbf{T})$. Лемма доказана.

Из доказательства леммы 1 следует, что

$$
\operatorname{dim} \operatorname{Ker}(H(k)-z I)=\operatorname{dim} \operatorname{Ker}(G(k, z)-I) .
$$

Теперь изучим, какими свойствами обладает предельный оператор $Q(k, m(k)) \equiv$ $Q(k)$. Пользуясь представлением

$$
\varepsilon_{k}(p)-m(k)=\frac{1}{4} \omega(k) \varepsilon_{0}(p)
$$

запишем ядро интегрального оператора $Q(k)$ в виде

$$
Q(k ; p, q)=\frac{v^{1 / 2}(p-q)}{\sqrt{\varepsilon_{k}(p)-m(k)}}=\frac{2}{\sqrt{\omega(k)}} \frac{v^{1 / 2}(p-q)}{\sqrt{\varepsilon_{0}(p)}} .
$$


Здесь $v^{1 / 2}(p-q)$ - ядро интегрального оператора $V^{1 / 2}$, оно равно

$$
v^{1 / 2}(p-q)=\sum_{n=1}^{\infty} \hat{v}^{1 / 2}(n) \varphi_{n}(p) \varphi_{n}(q),
$$

где $\varphi_{n}$ определяется по формуле (3.1). Из (3.7) и равенства $\omega(0)=4$ следует, что

$$
Q(k ; p, q)=\frac{2}{\sqrt{\omega(k)}} Q(0 ; p, q),
$$

откуда вытекает операторное равенство

$$
Q(k)=\frac{2}{\sqrt{\omega(k)}} Q(0) .
$$

Легко проверить, что действия оператора $Q(0)$ на элементы ортонормированного базиса $\left\{\varphi_{n}\right\}_{1}^{\infty}$ равны (см. (3.7) и (3.8))

$$
\left(Q(0) \varphi_{n}\right)(p)=\frac{\hat{v}^{1 / 2}(n) \varphi_{n}(p)}{\sqrt{\varepsilon_{0}(p)}} .
$$

Из (3.10) вытекает неравенство

$$
\left\|Q(0) \varphi_{n}\right\|^{2} \leqslant C \hat{v}(n) n
$$

при некотором $C>0$ и для всех $n \in \mathbb{N}$. При условии $(2.1)$ последовательность $\left\{\left\|Q(0) \varphi_{n}\right\|\right\} \in \ell_{2}$. Поэтому $Q(0)$ принадлежит классу Гильберта-Шмидта $\Sigma_{2}$. Отсюда и из $(3.9)$ следует, что $Q(k) \in \Sigma_{2}$ при каждом $k \in(-\pi, \pi)$. Можно показать, что при каждом $k \in(-\pi, \pi)$ оператор $Q(k, z)$ равномерно сходится к предельному оператору $Q(k) \equiv Q(k, m(k))$ при $z \rightarrow m(k)$. Для любого $k \in(-\pi, \pi)$ обозначим через $G(k, m(k)) \equiv G(k)$ положительный оператор $(Q(k))^{*} Q(k)$. Из равенства $(3.9)$ следует, что

$$
G(k) \equiv(Q(k))^{*} Q(k)=\frac{4}{\omega(k)} G(0) .
$$

Кроме того, $G(k) \in \Sigma_{1}$ для любого $k \in(-\pi, \pi)$. В работе [11] доказана более общая теорема, из которой следует

$$
N(k) \geqslant n(\omega(k), V)=n\left(4 \cos \frac{k}{2}, V\right),
$$

где правая часть - неубывающая функция по $k \in[0, \pi]$. Это наводит на мысль, что $N(k)$ также обладает этим свойством. Оказывается, это действительно так (см. ниже теорему 1). Сначала докажем, что принцип Бирмана-Швингера имеет место и в случае $z=m(k)$.

Лемма 2. Пусть выполнено условие (2.1). Тогда для любого $k \in(-\pi, \pi)$ имеет место равенство

$$
N(k)=n(1, G(k)) .
$$


ДоКАЗАтЕЛЬСТво. При условии (2.1) оператор $Q(k, z)$ принадлежит $\Sigma_{2}$ при всех $k \in(-\pi, \pi)$ и $z \leqslant m(k)$. Из представления $G(k, z)=(Q(k, z))^{*} Q(k, z)$ следует включение $G(k, z) \in \Sigma_{1}$. С учетом того, что $\|Q(k, m(k))-Q(k, z)\| \rightarrow 0$ при $z \rightarrow m(k)$, можно показать, что

$$
\lim _{z \rightarrow m(k)-}\|G(k, m(k))-G(k, z)\|=0 .
$$

Из компактности $G(k, m(k)) \equiv G(k)$ следует, что число $n(1, G(k))$ конечно. Для любого $\psi \in L_{2}^{-}(\mathbf{T})$ и $z<m(k)$ имеет место соотношение

$$
(G(k, z) \psi, \psi)=\int_{\mathbf{T}} \frac{\left|\left(V^{1 / 2} \psi\right)(p)\right|^{2} d p}{\varepsilon_{k}(p)-z} \leqslant \int_{\mathbf{T}} \frac{\left|\left(V^{1 / 2} \psi\right)(p)\right|^{2} d p}{\varepsilon_{k}(p)-m(k)}=(G(k) \psi, \psi) .
$$

Следовательно, мы имеем

$$
n(1, G(k, z)) \leqslant n(1, G(k)) .
$$

Пусть $\lambda_{1}(k) \geqslant \lambda_{2}(k) \geqslant \cdots \geqslant \lambda_{n}(k)>1$ - собственные значения оператора $G(k)$, $\psi_{1}, \psi_{2}, \ldots, \psi_{n}$ - соответствующие им собственные функции. Обозначим через $\mathcal{H}_{n}=$ $\operatorname{span}\left\{\psi_{1}, \psi_{2}, \ldots, \psi_{n}\right\}$ подпространство, натянутое на собственные функции $\psi_{1}$, $\psi_{2}, \ldots, \psi_{n}$. Выберем $\delta>0$ так, чтобы при всех $z \in(m(k)-\delta, m(k)]$ выполнялось неравенство

$$
\|G(k, z)-G(k)\|<\lambda_{n}(k)-1 .
$$

Тогда для любого ненулевого $\psi \in \mathcal{H}_{n}$ имеем

$$
(G(k, z) \psi, \psi)=(G(k) \psi, \psi)-([G(k)-G(k, z)] \psi, \psi)>(\psi, \psi),
$$

откуда следует, что

$$
n(1, G(k, z)) \geqslant \operatorname{dim} \mathcal{H}_{n}=n(1, G(k)) \quad \text { при } \quad z \in(m(k)-\delta, m(k)] .
$$

Комбинируя (3.14) и (3.16), получим

$$
n(1, G(k, z))=n(1, G(k)) \quad \text { при } \quad z \in(m(k)-\delta, m(k)] .
$$

Из принципа Бирмана-Швингера (3.2) при $z<m(k)$ и равенства (3.17) следует, что

$$
N(k, z)=n(1, G(k)) \quad \text { при } \quad z \in(m(k)-\delta, m(k)) .
$$

По определению $N(k, z)$ - кусочно-постоянная и непрерывная слева по $z \leqslant m(k)$ функция, поэтому имеет место (3.13). Лемма доказана.

Теперь изучим число собственных значений оператора $G(k)$. В силу равенства (3.11) достаточно изучить число собственных значений оператора $G(0)$. Собственные значения положительного компактного оператора $G(0)$ напишем в порядке убывания: $\mu_{1}>\mu_{2}>\cdots>\mu_{n}>\cdots$, и кратность собственного значения $\mu_{n}, \quad n \in \mathbb{N}$, обозначим через $\ell_{n}, n \in \mathbb{N}$. При выполнении условия $(2.3)$ оператор $G(0)$ имеет бесконечное число положительных собственных значений, поэтому $\mu_{n}>0$ для любого 
$n \in \mathbb{N}$. В силу компактности $G(0)$ имеем $\mu_{n} \rightarrow 0$ при $n \rightarrow \infty$. Из равенств (3.13) и (3.11) получим

$$
N(k)=n\left(1, \frac{4}{\omega(k)} G(0)\right)=\sum_{\mu_{n}>\omega(k) / 4} \ell_{n} .
$$

Отсюда следует, что точки разрыва $N(k)$ являются решением уравнения $4 \mu_{n}=\omega(k)$, т.е. уравнения

$$
\mu_{n}=\cos \frac{k}{2} .
$$

Если $\mu_{n} \in[0,1]$, то уравнение (3.19) имеет единственное решение $k_{n}=2 \arccos \mu_{n}$ на отрезке $[0, \pi]$. Из (3.18) следует, что скачок в точке разрыва $k_{n}$ равен $N\left(k_{n}+0\right)-$ $N\left(k_{n}\right)=\ell_{n}$. Таким образом, доказана следующая теорема.

Теорема 1. Число собственных значений $N(k)$ - неубывающая функция на $[0, \pi]$, она определяется по формуле (3.18), точки разрыва $N(k)$ есть $k_{n}=2 \arccos \mu_{n}$ и скачок в точке $k_{n}$ равен $\ell_{n}$. Если выполняется условие (2.3), то

$$
\lim _{k \rightarrow \pi-} N(k)=\infty .
$$

Заметим, что если оператор $V$ имеет ранг $n$, то (см. (3.12))

$$
N(k)=N(\pi)=n, \quad \text { если } \min _{s \in \operatorname{supp} \hat{v}}\{\hat{v}(s)\}>\omega(k)=4 \cos \frac{k}{2} .
$$

Теперь рассмотрим случай $k=k_{n}$. Из вышесказанного следует, что с уменьшением полного квазиимпульса $k$ некоторые собственные значения оператора $H(k)$ приближаются к краю непрерывного спектра и при $k=k_{n}$ попадают на край. При $k<k_{n}$ они исчезают, поглощаясь непрерывным спектром, и превращаются в резонансы.

Теорема 2. Пусть для некоторых $C>0$ и а >0 выполняется неравенство

$$
|\hat{v}(n)| \leqslant C e^{-a n} .
$$

Тогда существует $\delta>0$ такое, что при всех $k \in\left(k_{n}-\delta, k_{n}\right)$ оператор $H(k)$ имеет ровно $\ell_{n}$ резонансов, лежащих в некоторой є-окрестности $(\epsilon>0) U_{\epsilon}\left(m\left(k_{n}\right)\right)=$ $\left\{z \in \mathbb{C}:\left|m\left(k_{n}\right)-z\right|<\epsilon\right\}$ точки $m\left(k_{n}\right)$.

ДокАЗАТЕльСТво. При выполнении (3.20) функция $v(p)$ является аналитической в некоторой комплексной $\epsilon$-окрестности $(\epsilon>0)$ отрезка $[-\pi, \pi]$. В работе [12] доказано, что число собственных значений и резонансов оператора Фридрихса, лежащих в достаточно малой окрестности $U_{\epsilon}\left(m\left(k_{n}\right)\right)=\left\{z \in \mathbb{C}:\left|m\left(k_{n}\right)-z\right|<\epsilon\right\}$, не меняется при малых возмущениях. Далее, применяя процедуру, аналогичную той, которая была использована в работе [12], можно завершить доказательство теоремы.

Теперь обсудим следующий вопрос: когда левый край $m(k)$ непрерывного спектра будет собственным значением оператора $H(k)$, т.е. при каких условиях решение $f$ уравнения Шредингера (3.3) при $z=m(k)$ принадлежит $L_{2}^{-}(\mathbf{T})$ ? Пусть уравнение $G(k) \psi=\psi$ имеет нетривиальное решение $\varphi \in L_{2}^{-}(\mathbf{T})$. С помощью соотношений (3.4) можно показать, что функция

$$
f(p)=\frac{\left(V^{1 / 2} \varphi\right)(p)}{\varepsilon_{0}(p)}
$$


удовлетворяет уравнению Шредингера (3.3) при $z=m(k)$. Если дополнительно

$$
V^{1 / 2} \varphi \in C^{1+1 / 2+0}(\mathbf{T}) \equiv\left\{g \in C^{(1)}(\mathbf{T}):\left|g^{\prime}(p)\right| \leqslant C|p|^{1 / 2+\epsilon} \quad \text { для некоторого } \epsilon>0\right\},
$$

то функция $f$, определенная в $(3.21)$, принадлежит $L_{2}^{-}(\mathbf{T})$.

При $z=m(k)$ равенство (3.6), вообще говоря, не имеет места. Например, если $\mu_{n} \in(0,1]$ - простое собственное значение оператора $G(0)$, а соответствующая собственная функция $\varphi$ такая, что $\left(V^{1 / 2} \varphi\right)^{\prime}(0) \neq 0$, тогда

$$
\operatorname{dim} \operatorname{Ker}(H(k)-m(k) I)=0<1=\operatorname{dim} \operatorname{Ker}(G(k)-I) .
$$

Равенство (3.6) при $z=m(k)$ превращается в неравенство

$$
\operatorname{dim} \operatorname{Ker}(H(k)-m(k) I) \leqslant \operatorname{dim} \operatorname{Ker}(G(k)-I) \text {. }
$$

\section{4. МОНОТОННОСТЬ СОБСТВЕННЫХ ЗНАЧЕНИЙ}

Докажем монотонность каждого собственного значения $z_{n}(k)$ в сформулированном ниже предположении. Обозначим через $\lambda_{1}(k, z) \geqslant \lambda_{2}(k, z) \geqslant \cdots \geqslant \lambda_{n}(k, z)>0$ первые $n$ собственных значений оператора $G(k, z)$ и через $\mathcal{H}_{n}(G(k, z))=$ $\operatorname{span}\left\{\psi_{1}, \psi_{2}, \ldots, \psi_{n}\right\}$ подпространство, натянутое на соответствующие собственные функции $\psi_{1}, \psi_{2}, \ldots, \psi_{n}$. Так как $G(-k, z)=G(k, z)$, то каждое собственное значение $\lambda_{n}(k, z)$ - четная функция по $k \in[-\pi, \pi]$. Теперь докажем их монотонность по $z \in(-\infty, m(k))$ и $k \in[0, \pi]$.

Лемма 3. Для любого $k \in \mathbf{T}$ каждое положительное собственное значение $\lambda_{n}(k, z)$ оператора $G(k, z)$ возрастает nо $z \in(-\infty, m(k))$.

ДоказАтЕльСтво. Для любого $\psi \in \mathcal{H}_{n}\left(G\left(k, z_{1}\right)\right)=\operatorname{span}\left\{\psi_{1}, \psi_{2}, \ldots, \psi_{n}\right\}$, отличного от нуля, и $z_{1}<z_{2} \in(-\infty, m(k))$ имеет место неравенство

$$
\left(G\left(k, z_{1}\right) \psi, \psi\right)=\int_{\mathbf{T}} \frac{\left|\left(V^{1 / 2} \psi\right)(p)\right|^{2} d p}{\varepsilon_{k}(p)-z_{1}}<\int_{\mathbf{T}} \frac{\left|\left(V^{1 / 2} \psi\right)(p)\right|^{2} d p}{\varepsilon_{k}(p)-z_{2}}=\left(G\left(k, z_{2}\right) \psi, \psi\right) .
$$

Поэтому $\lambda_{n}\left(k, z_{1}\right)<\lambda_{n}\left(k, z_{2}\right)$. Лемма доказана.

ПРЕДПОЛОЖЕНИЕ. Пусть либо

$$
\operatorname{supp} \hat{v} \subset\{2 n: n \in \mathbb{Z}\}
$$

либо

$$
\operatorname{supp} \hat{v} \subset\{2 n+1: n \in \mathbb{Z}\} .
$$

ЛЕмма 4. Пусть выполнено наше предположение $u z<m\left(k_{0}\right)$. Тогда каждое положительное собственное значение $\lambda_{n}(k, z)$ оператора $G(k, z)$ yбывает по $k \in$ $\left[k_{0}, \pi\right]$.

ДокАЗАТЕЛЬСтво. При выполнении условия (4.1) функция $v^{1 / 2}(p)$ является $\pi$ периодической, а при выполнении (4.2) - $\pi$-антипериодической, т.е.

$$
v^{1 / 2}(p+\pi)=-v^{1 / 2}(p) .
$$


Следовательно, в обоих случаях при всех $\psi \in L_{2}^{-}(\mathbf{T})$ функция $\left|\left(V^{1 / 2} \psi\right)(p)\right|=|\varphi(p)|$ является $\pi$-периодической. Теперь рассмотрим квадратичную форму

$$
(G(k, z) \psi, \psi)=\int_{-\pi}^{\pi} \frac{|\varphi(p)|^{2} d p}{2-2 \cos (k / 2) \cos p-z} .
$$

Интеграл представим как сумму двух интегралов по отрезкам $[-\pi, 0]$ и $[0, \pi]$. Сделав замену переменной $p^{\prime}=\pi+p$ в первом интеграле и использовав тождества $\cos (\pi+$ $p)=-\cos p$ и $|\varphi(p+\pi)|=|\varphi(p)|$, имеем

$$
(G(k, z) \psi, \psi)=2(2-z) \int_{0}^{\pi} \frac{|\varphi(p)|^{2} d p}{(2-z)^{2}-4 \cos ^{2}(k / 2) \cos ^{2} p} .
$$

Так как $2-z>0$ при $z<m\left(k_{0}\right)$, а интеграл в (4.3) убывает по $k \in\left[k_{0}, \pi\right]$, то для любого ненулевого $\psi \in \mathcal{H}_{n}(G(k, z))$ имеет место неравенство

$$
(G(k, z) \psi, \psi)>\left(G\left(k^{\prime}, z\right) \psi, \psi\right) \quad \text { при } \quad k_{0} \leqslant k<k^{\prime} \leqslant \pi .
$$

Это доказывает, что $\lambda_{n}(k, z)>\lambda_{n}\left(k^{\prime}, z\right)$ при $k_{0} \leqslant k<k^{\prime} \leqslant \pi$. Лемма доказана.

Из теоремы 1 следует, что если при некотором $k \in[0, \pi]$ существует собственное значение $z_{n}(k)$, то оно не исчезает при возрастании полного квазиимпульса. Поэтому область определения собственного значения $z_{n}(k)$ имеет вид $\left[-\pi,-k_{n}\right) \cup\left(k_{n}, \pi\right]$. Если оператор $G(0)$ имеет $m$ собственных значений, больших 1 с учетом кратности, то область определения собственных значений $z_{1}(k) \leqslant z_{2}(k) \leqslant \cdots \leqslant z_{m}(k)$ совпадает c $\mathbf{T}$.

ТЕОРема 3. Пусть выполнено наше предположение. Тогда каждое собственное значение $z_{n}(k)$ оператора $H(k)$ возрастает nо $k \in\left(k_{n}, \pi\right]$.

ДокАЗАТЕЛЬСтво. Пусть $k$ и $k^{\prime}$ - две произвольные точки такие, что $k_{n}<$ $k<k^{\prime} \leqslant \pi$. Пусть оператор $H(k)$ имеет $N=N(k)$ собственных значений $z_{1}(k) \leqslant$ $z_{2}(k) \leqslant \cdots \leqslant z_{N}(k)$, лежащих ниже $m(k)$. Существование не менее $N(k)$ собственных значений оператора $H\left(k^{\prime}\right)$ следует из теоремы 1. Из (3.2) следует, что оператор $G(k, z)$ имеет ровно $N(k)$ собственных значений

$$
\lambda_{1}(k, z) \geqslant \lambda_{2}(k, z) \geqslant \cdots \geqslant \lambda_{N}(k, z)>1
$$

при $z \in\left(z_{N}(k), m(k)\right]$. Из непрерывности $G(k, z)$ следует непрерывность каждого $\lambda_{n}(k, z)$ по $z \in(-\infty, m(k))$. Легко показать, что

$$
\lim _{z \rightarrow-\infty}\|G(k, z)\|=0 .
$$

Согласно (4.4), (4.5) и неравенству $\lambda_{n}(k, z) \leqslant\|G(k, z)\|$ при любом $n \in\{1,2, \ldots, N\}$ уравнение $\lambda_{n}(k, z)=1$ имеет единственное решение $z=z_{n}(k) \in(-\infty, m(k))$. Единственность следует из возрастания $\lambda_{n}(k, \cdot)$. В силу леммы 1 число $z_{n}(k)$ является собственным значением оператора $H(k)$. Из неравенства $\lambda_{n}(k, z) \geqslant \lambda_{n+1}(k, z)$ и монотонности функции $\lambda_{n}(k, \cdot)$ следует, что $z_{n}(k) \leqslant z_{n+1}(k), \quad n=\overline{1, N-1}$. Теперь покажем возрастание $z_{n}(k)$ по $k \in\left(k_{n}, \pi\right]$. В силу леммы 4 собственное значение $\lambda_{n}(k, z)$ - убывающая функция по $k \in\left(k_{n}, \pi\right]$. Поэтому

$$
1=\lambda_{n}\left(k, z_{n}(k)\right)>\lambda_{n}\left(k^{\prime}, z_{n}(k)\right) .
$$


С другой стороны,

$$
1=\lambda_{n}\left(k^{\prime}, z_{n}\left(k^{\prime}\right)\right)>\lambda_{n}\left(k^{\prime}, z_{n}(k)\right) .
$$

Так как $\lambda_{n}(k, \cdot)$ - возрастающая функция, то $z_{n}\left(k^{\prime}\right)>z_{n}(k)$. Теорема доказана.

Отметим, что выполнение сформулированного выше предположения является существенным.

ПримеР. Пусть потенциал $\hat{v}$ удовлетворяет условию

$$
\hat{v}(1)>\hat{v}(2)>\cdots>\hat{v}(n)>\cdots .
$$

Тогда оператор $H(\pi)=2 I-V$ имеет простые собственные значения вида $z_{n}(\pi)=$ $2-\hat{v}(n), \quad n \in \mathbb{N}$. Применяя теорему Реллиха (см. [1]) для оператора $H(k)=H(\pi)-$ $\cos (k / 2) W$, где $W$ - оператор умножения на функцию $2 \cos p$, мы утверждаем, что оператор $H(k)$ имеет единственное простое собственное значение $z_{n}(k)$ в окрестности $z_{n}(\pi)$ при малых $\pi-k$. Несложные вычисления показывают, что ряд РэлеяШредингера для $z_{n}(k)$ при $n \geqslant 2$ имеет вид

$$
z_{n}(k)=z_{n}(\pi)+\frac{(\Delta \hat{v})(n)(\cos (k / 2))^{2}}{(\hat{v}(n)-\hat{v}(n-1))(\hat{v}(n)-\hat{v}(n+1))}+\sum_{s=2}^{\infty} C_{s}\left(\cos \frac{k}{2}\right)^{2 s} .
$$

Если выполняется условие (4.6), то знаменатель второго слагаемого в (4.7) отрицателен. Если, кроме того, $(\Delta \hat{v})(n)<0$, то существует $\delta>0$ такое, что собственное значение $z_{n}(k), n \geqslant 2$, убывает на отрезке $[\pi-\delta, \pi]$.

Благодарности. Работа частично финансирована ФПФИ АН РУз (грант № 67-04).

\section{Список литературы}

[1] М. Рид, Б. Саймон, Методы современной математической физики. T. IV. Анализ операторов, Мир, М., 1982.

[2] J. Rauch, J. Funct. Anal., 35 (1980), 304-315.

[3] B. Simon, Ann. Phys., 97 (1976), 279-288.

[4] M. Klaus, Ann. Phys., 108 (1977), 288-300.

[5] J. S. Howland, Pacific J. Math., 55 (1974), 157-176.

[6] Ш. С. Маматов, Р. А. Минлос, ТМФ, 79:2 (1989), 163-179.

[7] R. A. Minlos, A. I. Mogilner, "Some problems concerning spectra of lattice models", Schrödinger Operators: Standard and Nonstandard, eds. P. Exner, P. Seba, World Scientific, Singapore, 1989, 243-257.

[8] T. Regge, Nuovo Cimento, 8 (1958), 671-679.

[9] П. Лакс, Р. С. Филлипс, Теория рассеяния, Мир, М., 1971.

[10] P. A. Faria da Viega, L. Ioriatti, M. O'Carrol, Phys. Rev. E, 66 (2002), 016130; D. C. Mattis, Rev. Mod. Phys., 58:2 (1986), 361-379.

[11] S. Albeverio, S. N. Lakaev, J. I. Abdullaev, On the spectral properties of two-particle discrete Schrödinger operators, Preprint, Germany, Universität Bonn, SFB 611, № 170, 2004.

[12] J. I. Abdullaev, S. N. Lakaev, Adv. Sov. Math., 5 (1991), 1-37.

Поступила в редакцию 6.VI.2005 г. 